\title{
Crystal structure of ipratropium bromide monohydrate, $\mathrm{C}_{20} \mathrm{H}_{30} \mathrm{NO}_{3} \mathrm{Br}\left(\mathrm{H}_{2} \mathrm{O}\right)$
}

\author{
Shivang Bhaskar, ${ }^{1}$ Joseph T. Golab, ${ }^{1}$ James A. Kaduk $\mathbb{\oplus},{ }^{2,3, a)}$ Amy M. Gindhart, ${ }^{4}$ and \\ Thomas N. Blanton $\mathbb{D}^{4}$ \\ ${ }^{I}$ Illinois Mathematics and Science Academy, 1500 Sullivan Rd., Aurora, Illinois 60506-1000, USA \\ ${ }^{2}$ Illinois Institute of Technology, 3101 S. Dearborn St., Chicago, Illinois 60616, USA \\ ${ }^{3}$ North Central College, 131 S. Loomis St., Naperville, Illinois 60540, USA \\ ${ }^{4}$ ICDD, 12 Campus Blvd., Newtown Square, Pennsylvania 19073-3273, USA
}

(Received 4 December 2019; accepted 27 December 2019)

\begin{abstract}
The crystal structure of ipratropium bromide monohydrate has been solved and refined using synchrotron X-ray powder diffraction data and optimized using density functional techniques. Ipratropium bromide monohydrate crystallizes in the space group $P 2_{1} / c$ (\#14) with $a=8.21420(7) \AA, b=$ $10.54617(13) \AA, c=24.0761(39) \AA, \beta=99.9063(7)^{\circ}, V=2054.574(22) \AA^{3}$, and $Z=4$. Both hydrogen atoms of the water molecule act as donors to the bromide cation, forming a ring with the graph set $R 2,4(8)$. The hydroxyl group also acts as a donor to $\mathrm{Br}$. Several $\mathrm{C}-\mathrm{H} \cdots \mathrm{Br}$ hydrogen bonds are present. The water molecule acts as an acceptor in two $\mathrm{C}-\mathrm{H} \cdots \mathrm{O}$ hydrogen bonds from methyl groups. The ketone acts as an acceptor in $\mathrm{C}-\mathrm{H} \cdots \mathrm{O}$ hydrogen bonds from methyl groups, a methylene group, and a methyne group. The hydroxyl group acts as an acceptor in a $\mathrm{C}-\mathrm{H} \cdots \mathrm{O}$ hydrogen bond from a phenyl carbon atom. The powder pattern is included in the Powder Diffraction File ${ }^{\mathrm{TM}}$ as entry 00-066-1611. (c) 2020 International Centre for Diffraction Data.

[doi:10.1017/S0885715620000020]
\end{abstract}

Key words: ipratropium bromide monohydrate, Atrovent ${ }^{\circledR}$, powder diffraction, Rietveld refinement, density functional theory

\section{INTRODUCTION}

Ipratropium bromide (brand names include: Atrovent, Apovent, Ipraxa, Aerovent, and Rinatec) is a drug which relieves bronchial spasms. The IUPAC name of ipratropium bromide monohydrate (CAS Registry number 66985-17-9) is (8-methyl-8-propan-2-yl-8-azoniabicyclo[3.2.1]octan-3-yl) 3-hydroxy-2-phenylpropanoate bromide monohydrate. A twodimensional molecular diagram for the ipratropium bromide cation is shown in Figure 1. It is on the World Health Organization's List of Essential Medicines, a listing of the most important medications needed in a basic health system. Ipratropium bromide is the bromide salt form of Ipratropium. Ipratropium bromide actively binds to cholinergic receptors in the bronchi, preventing constriction of the airway. This leads to the dilation of the airways, which induces bronchodilation.

Low-precision powder patterns of ipratropium bromide monohydrate are present in the Powder Diffraction File as entries 00-043-1754 (Bernstein and Zevin, 1992) and 00-058-1427 (Abdine et al., 2003). Powder patterns of native and spray-dried ipratropium bromide are reported in Corrigan et al. (2006).

This work was carried out as part of a project (Kaduk et al., 2014) to determine the crystal structures of largevolume commercial pharmaceuticals, and include high-quality powder diffraction data for these pharmaceuticals in the Powder Diffraction File (Gates-Rector and Blanton, 2019).

\footnotetext{
a) Author to whom correspondence should be addressed. Electronic mail: kaduk@polycrystallography.com
}

\section{EXPERIMENTAL}

Ipratropium bromide monohydrate was a commercial reagent, purchased from the United States Pharmacopeial Convention (USP) (Lot \#R022A0), and was used as-received. The white powder was packed into a 1.5-mm-diameter Kapton capillary and rotated during the measurement at $\sim 50 \mathrm{~Hz}$. The powder pattern was measured at $295 \mathrm{~K}$ at beamline 11-BM (Lee et al., 2008; Wang et al., 2008) of the Advanced Photon Source at the Argonne National Laboratory using a wavelength of $0.413342 \AA$ from 0.5 to $50^{\circ} 2 \theta$ with a step size of $0.001^{\circ}$ and a counting time of $0.1 \mathrm{~s} \mathrm{step}^{-1}$.

The pattern was indexed on a primitive monoclinic unit cell with $a=8.21411 \AA, b=10.54608 \AA, c=24.07614 \AA$, $\beta=99.905^{\circ}, V=2054.548 \AA^{3}$, and $Z=4$ using N-TREOR (Altomare et al., 2013). The analysis of the systematic absences using EXPO2014 (Altomare et al., 2013) suggested that the space group was $P 2_{1} / c$, which was confirmed by the successful solution and refinement of the structure. A reduced cell search in the Cambridge Structural Database (Groom et al., 2016) with the chemistry $\mathrm{C}, \mathrm{H}, \mathrm{N}, \mathrm{O}$, and $\mathrm{Br}$ only yielded no hits. An ipratropium cation was built using Spartan '18 (Wavefunction, 2018) and converted into a . mol2 file using OpenBabel (O'Boyle et al., 2011). The structure was solved by Monte Carlo simulated annealing techniques using FOX (Favre-Nicolin and Černý, 2002), using an ipratropium cation, and $\mathrm{Br}$ and $\mathrm{O}$ atom fragments.

Rietveld refinement was carried out using GSAS-II (Toby and Von Dreele, 2013). Only the $1.8-25.0^{\circ}$ portion of the pattern was included in the refinement $\left(d_{\min }=0.955 \AA\right)$. All non-H bond distances and angles were subjected to restraints, based on a Mercury/Mogul Geometry Check (Bruno et al., 


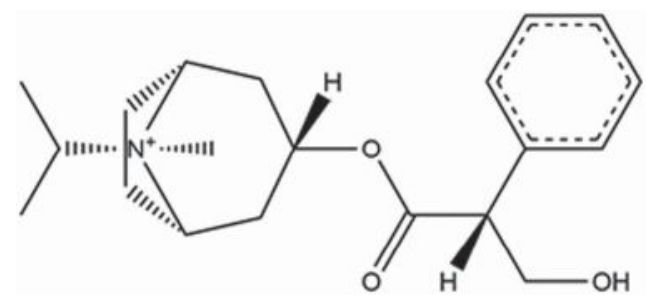

Figure 1. Molecular structure of the ipratropium cation.
2004; Sykes et al., 2011) of the molecule. The results were exported to a csv file. The Mogul average and standard deviation for each quantity were used as the restraint parameters and were incorporated using the new feature Restraints/Edit Restraints/Add MOGUL Restraints, which reads the bond distance and angle restraints from the csv file. The restraints contributed $2.2 \%$ to the final $\chi^{2}$. The hydrogen atoms were included in calculated positions, which were recalculated during the refinement using the Materials Studio (Dassault Systèmes, 2018). A common $U_{\text {iso }}$ was refined for the carbon

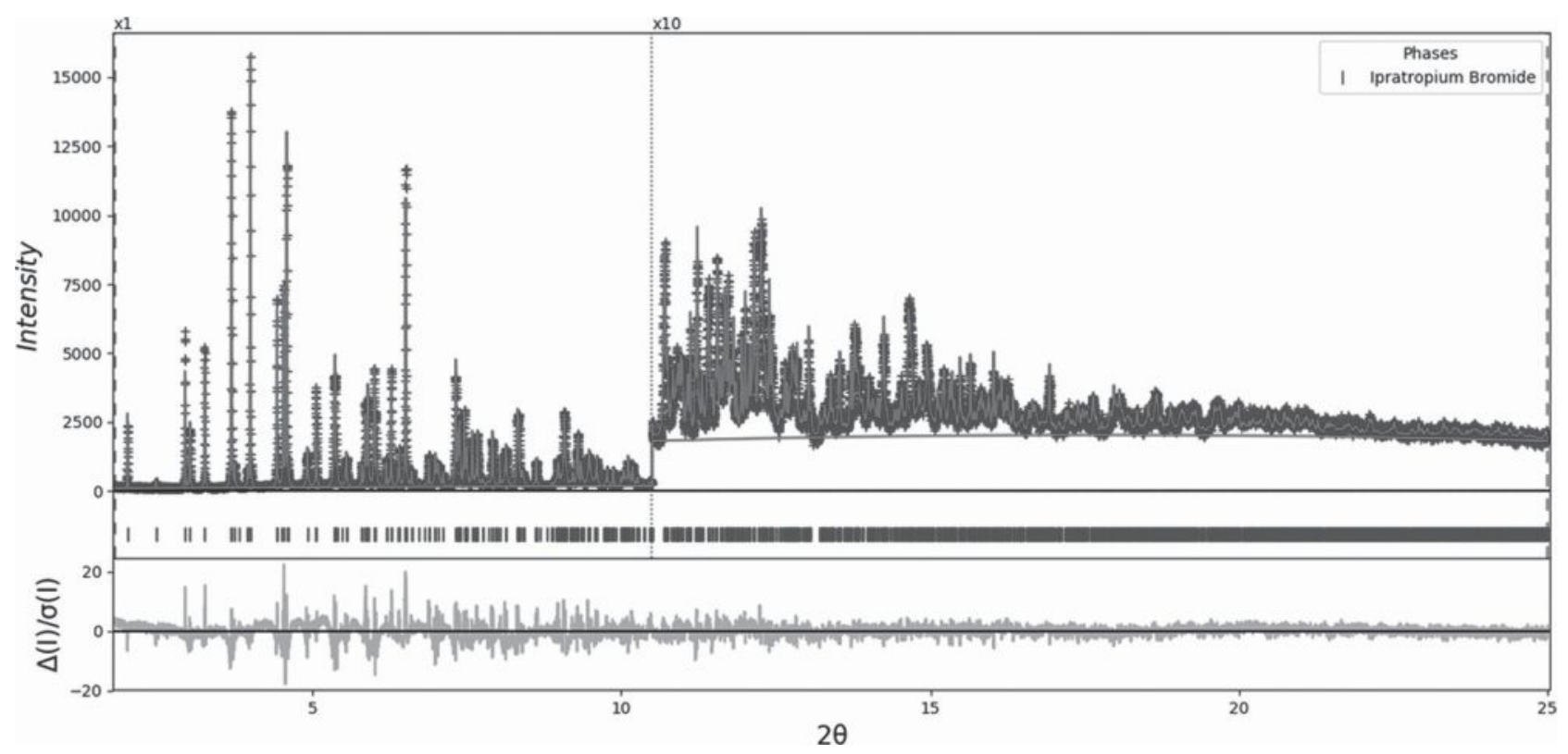

Figure 2. Rietveld plot for the refinement of ipratropium bromide monohydrate. The blue crosses represent the observed data points, and the green line is the calculated pattern. The cyan curve is the normalized error plot. The vertical scale has been multiplied by a factor of $10 \times$ for $2 \theta>10.5^{\circ}$.

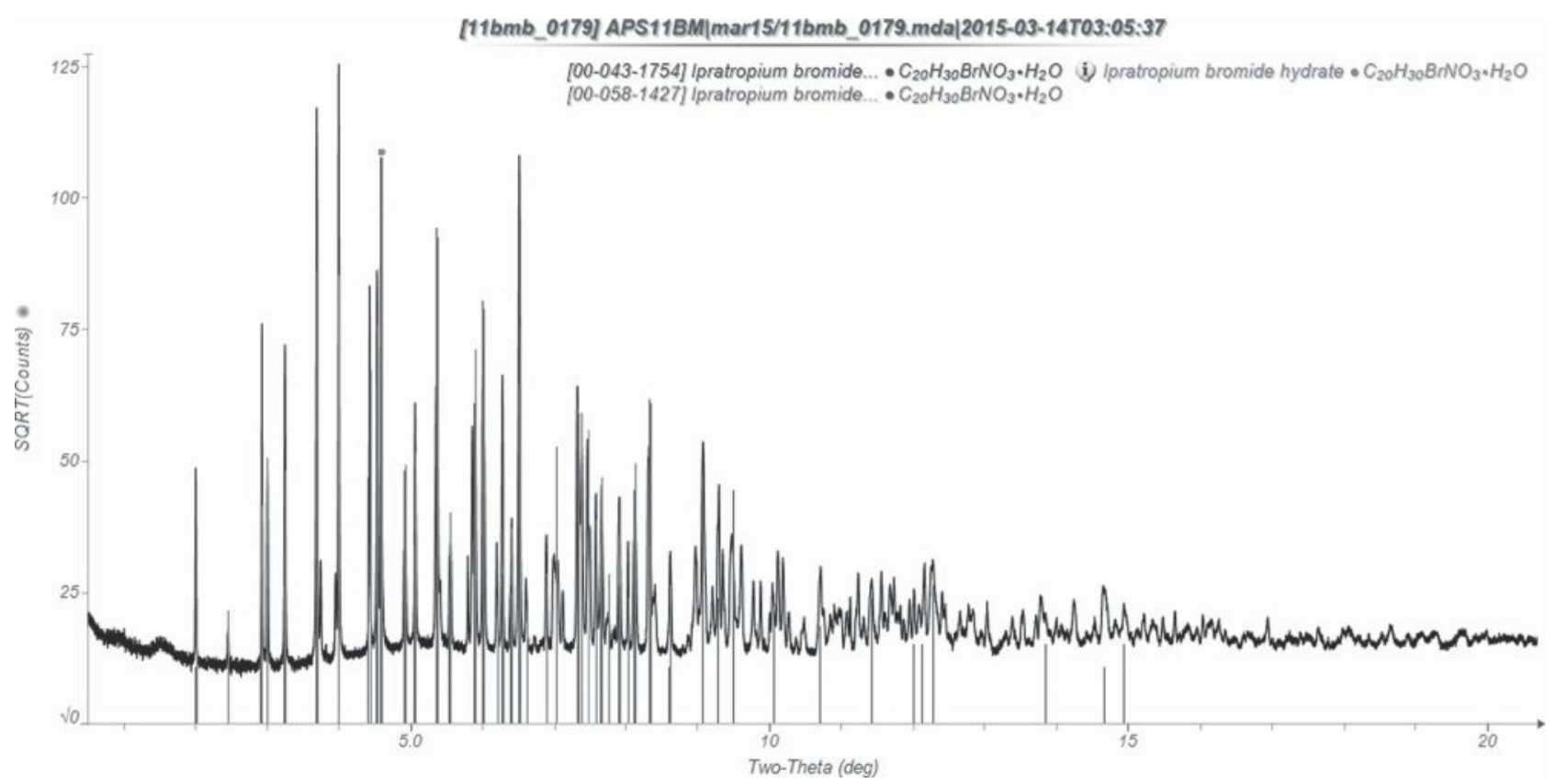

Figure 3. Comparison of the synchrotron pattern of this study to the two existing PDF entries (00-043-1754 and 00058-1427) for ipratropium bromide monohydrate. 


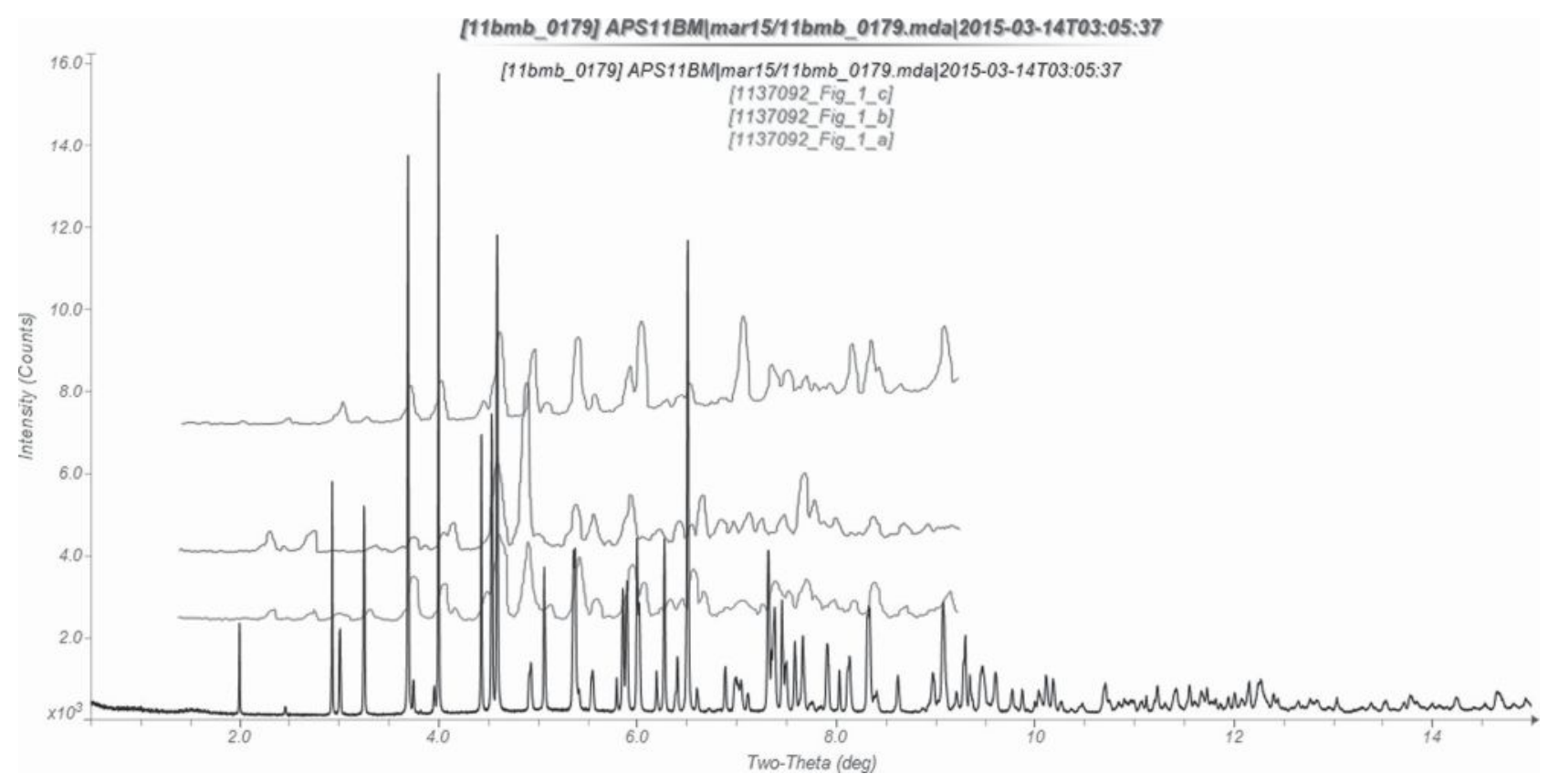

Figure 4. Comparison of the ipratropium bromide monohydrate powder pattern of this study to those reported in Corrigan et al. (2006). Figure 1(c) is the ipratropium bromide monohydrate starting material, while Figures 1(a) and 1(b) are patterns of spray-dried materials.

atoms of the phenyl ring, another $U_{\text {iso }}$ for the atoms of the cage, and the third $U_{\text {iso }}$ for the intermediate part of the molecule. We refined the bromine atom anisotropically. The $U_{\text {iso }}$ for each hydrogen atom was constrained to be $1.3 \times$ that of the heavy atom to which it is attached. The background was modeled using a 3-term shifted Chebyshev polynomial.

The final refinement of 105 variables using 23203 observations and 64 restraints yielded the residuals $R \mathrm{wp}=0.1171$ and $\mathrm{GOF}=2.35$. The largest peak $(0.40 \AA$ from $\mathrm{Br} 55)$ and hole $(1.07 \AA$ from $\mathrm{Br} 55)$ in the difference Fourier map were 0.67 and $-0.41(9) e \AA^{-3}$. The Rietveld plot is included in Figure 2. The largest errors in the fit are in the shapes of some of the strong low angle peaks.

A density functional geometry optimization was carried out using CRYSTAL14 (Dovesi et al., 2014). The basis sets for the $\mathrm{H}, \mathrm{C}, \mathrm{N}$, and $\mathrm{O}$ atoms were those of Gatti et al. (1994), and the basis set for $\mathrm{Br}$ was that of Peintinger et al. (2013). The calculation was run on eight $2.1 \mathrm{GHz}$ Xeon cores (each with 6 GB RAM) of a 304-core Dell Linux cluster at IIT, using eight $k$-points and the B3LYP functional, and took $\sim 110 \mathrm{~h}$.

\section{RESULTS AND DISCUSSION}

The powder pattern of ipratropium bromide monohydrate measured in this study is similar enough to PDF entries 00-043-1754 and 00-058-1427 to conclude that they represent the same material (Figure 3). Our pattern is most similar to the ipratropium bromide monohydrate starting material of Corrigan et al. (2006) and less similar to their spray-dried materials (Figure 4).

The refined atom coordinates of ipratropium bromide monohydrate and the coordinates from the density functional theory (DFT) optimization are reported in the CIFs. The root-mean-square (rms) Cartesian displacement of the non-H atoms in the Rietveld-refined and DFT-optimized structures

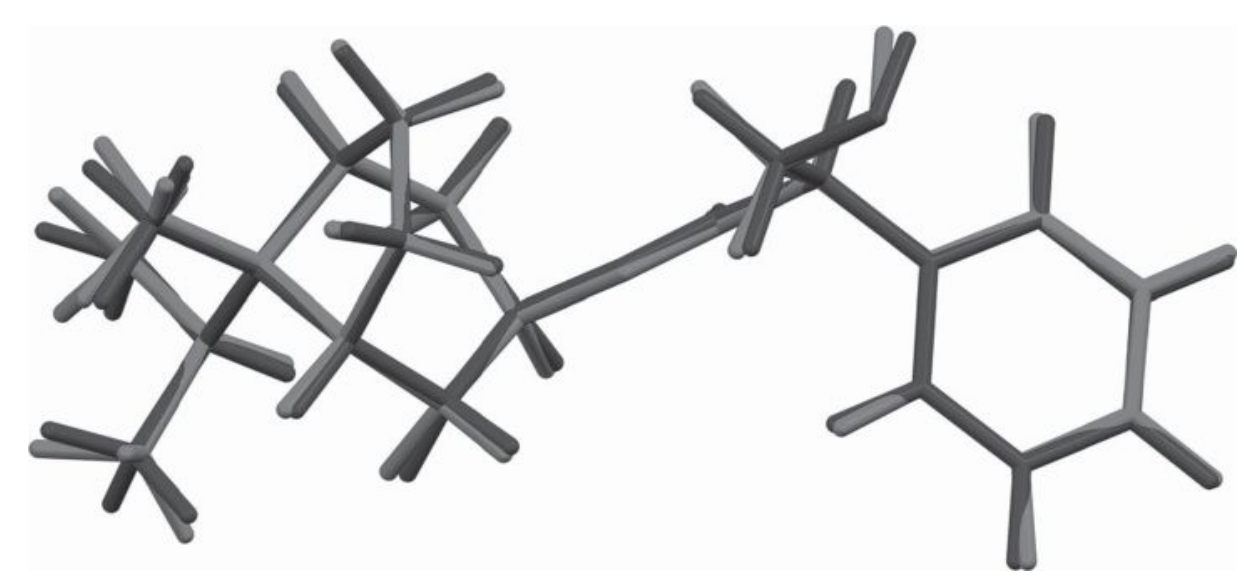

Figure 5. Comparison of the Rietveld-refined (red) and VASP-optimized (blue) structures of ipratropium bromide monohydrate. The rms Cartesian displacement is $0.075 \AA$. 


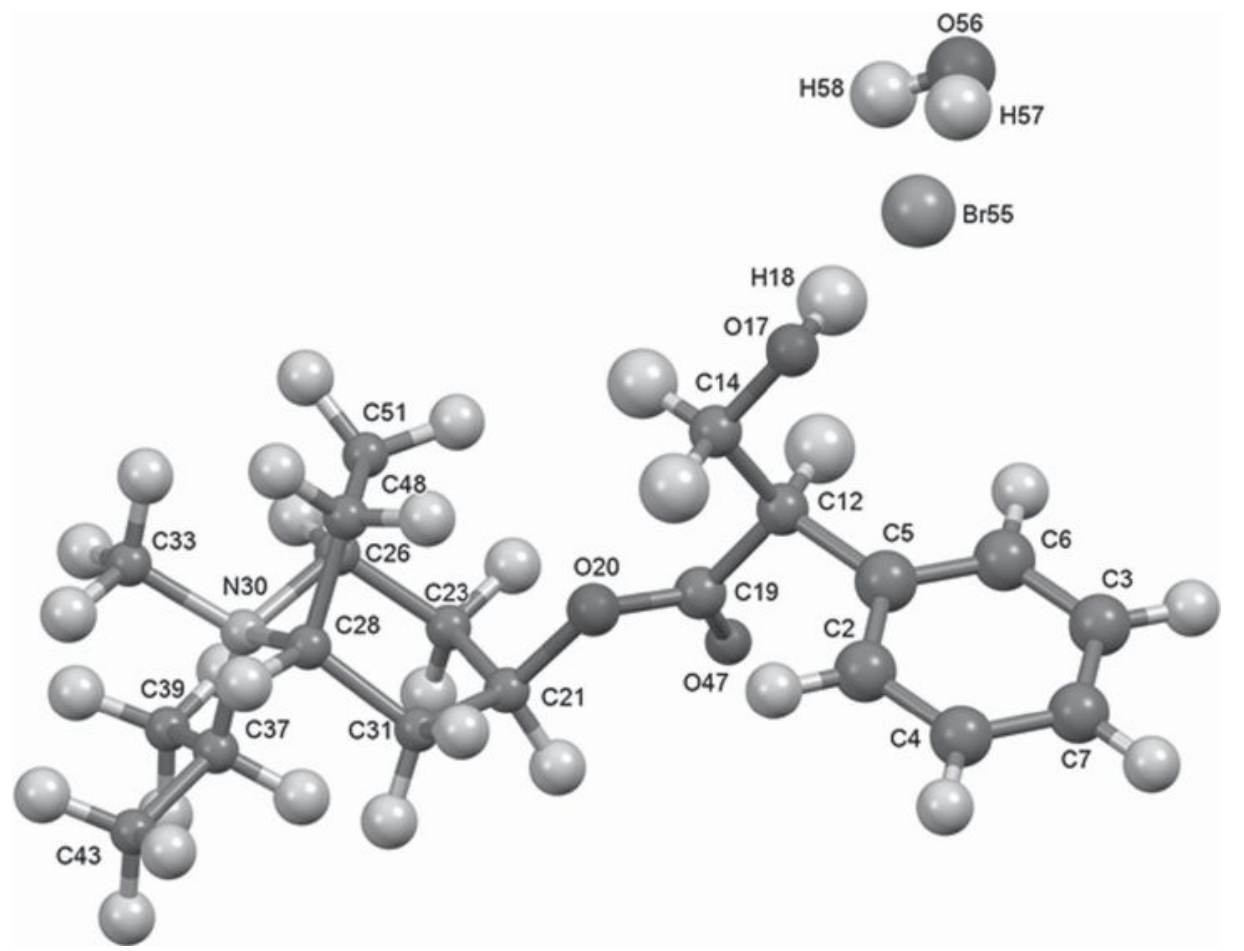

Figure 6. Asymmetric unit of ipratropium bromide monohydrate, with the atom numbering. The atoms are represented by $50 \%$ probability spheroids/ellipsoids.

is $0.075 \AA$ (Figure 5), and the maximum displacement is $0.208 \AA$. The excellent agreement between the refined and optimized structures provides evidence that the experimental structure is correct (van de Streek and Neumann, 2014). This discussion concentrates on the CRYSTAL-optimized structure. The asymmetric unit (with the atom numbering) is illustrated in Figure 6, and the crystal structure is presented in Figure 7. The crystal structure consists of layers parallel to the $a b$-plane. The cation-anion and hydrogen-bonding interactions occur within the layer, and between the layers are van der Waals interactions.

All of the bond distances and bond angles, and most of the torsion angles fall within the normal ranges indicated by a Mercury/Mogul Geometry Check (Macrae et al., 2008). The

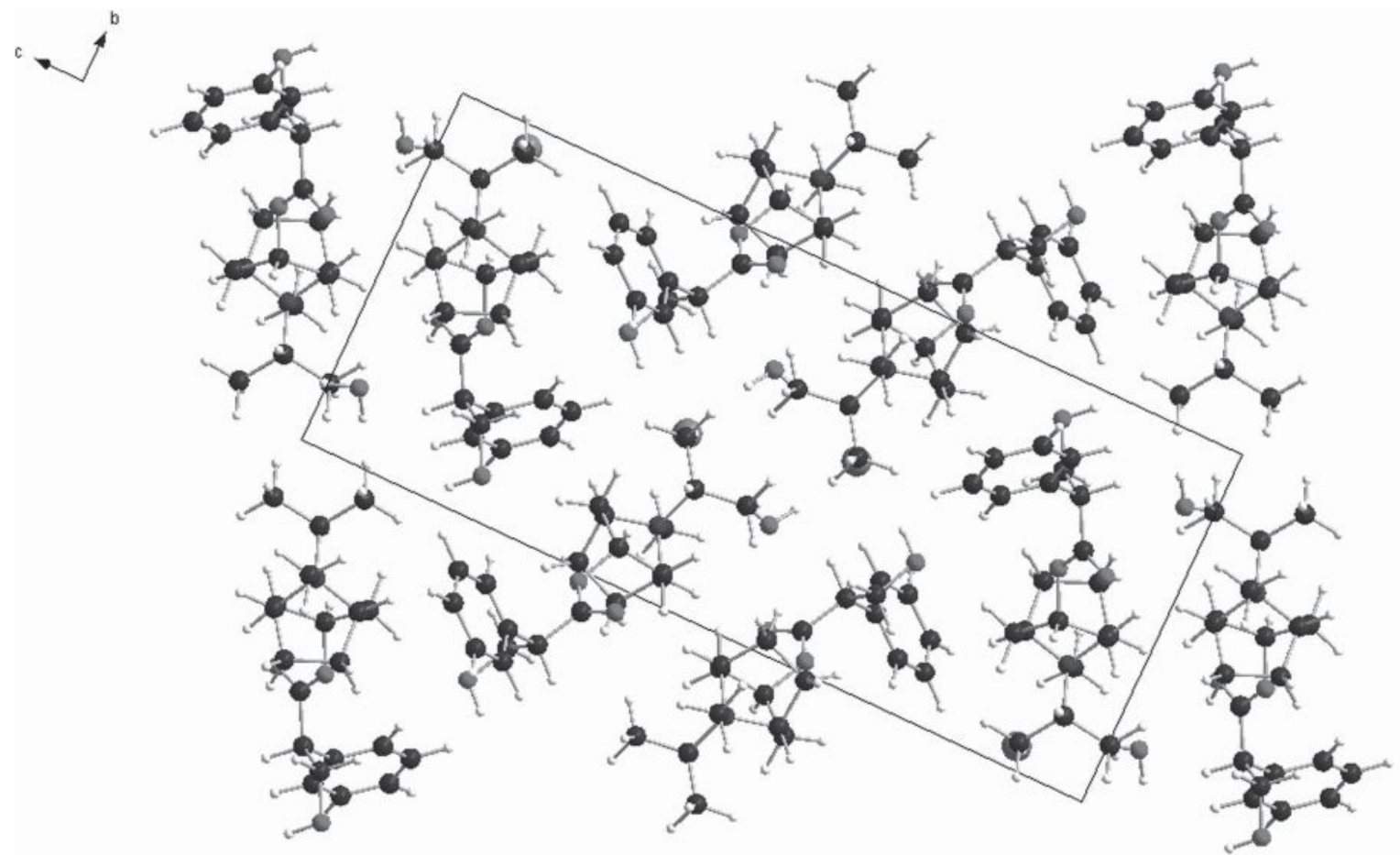

Figure 7. Crystal structure of ipratropium bromide monohydrate, viewed down the $a$-axis. 
TABLE I. Hydrogen bonds (CRYSTAL14) in ipratropium bromide monohydrate.

\begin{tabular}{|c|c|c|c|c|c|}
\hline H-bond & D-H $(\AA)$ & $\mathrm{H} \cdots \mathrm{A}(\AA)$ & $\mathrm{D} \cdots \mathrm{A}(\AA)$ & $\mathrm{D}-\mathrm{H} \cdots \mathrm{A}\left({ }^{\circ}\right)$ & Overlap $(e)$ \\
\hline 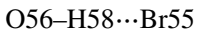 & 0.976 & 2.508 & 3.481 & 174.9 & 0.026 \\
\hline 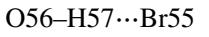 & 0.976 & 2.429 & 3.395 & 170.3 & 0.026 \\
\hline O17-H18‥Br55 & 0.977 & 2.434 & 3.395 & 167.6 & 0.030 \\
\hline 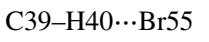 & 1.093 & 2.901 & 3.908 & 153.3 & 0.010 \\
\hline 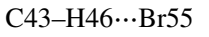 & 1.093 & 3.205 & 4.272 & 165.5 & 0.010 \\
\hline C31-H54‥Br55 & 1.093 & 2.866 & 3.796 & 143.0 & 0.013 \\
\hline 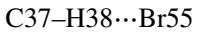 & 1.087 & 2.781 & 3.746 & 147.8 & 0.012 \\
\hline C43-H45‥O56 & 1.094 & 2.645 & 3.725 & 169.2 & 0.021 \\
\hline 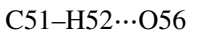 & 1.765 & 2.765 & 3.782 & 155.0 & 0.011 \\
\hline 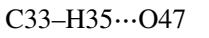 & 1.085 & 2.273 & 3.298 & 156.7 & 0.021 \\
\hline C39-H42 ‥ 447 & 1.093 & 2.659 & 3.750 & 176.0 & 0.013 \\
\hline 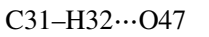 & 1.094 & 2.347 & 3.366 & 154.3 & 0.024 \\
\hline $\mathrm{C} 21-\mathrm{H} 22 \cdots \mathrm{O} 47$ & 1.090 & $2.448^{\mathrm{a}}$ & 2.707 & 91.6 & 0.010 \\
\hline C3-H11O17 & 1.087 & 2.774 & 3.718 & 145.0 & 0.015 \\
\hline
\end{tabular}

${ }^{\mathrm{a}}$ Intramolecular.

O17-C14-C12-C19 torsion angle of $178^{\circ}$ is flagged as unusual; it is part of a minor distribution of trans angles in a major population of gauche torsions. The O20-C19-C12C14 and O47-C19-C12-C14 torsions are also flagged as unusual; these are both part of a minor population in wider distributions. All three of these torsion angles involve rotation of the hydroxymethylphenyl portion of the molecule with respect to the rest of the cation. The conformation of the cation is unusual but not unprecedented.

Quantum chemical geometry optimization (DFT/B3LYP/ 6-31G*/water) using Spartan '18 (Wavefunction, 2018) indicated that the observed conformation of the ipratropium cation is $1.2 \mathrm{kcal} \mathrm{mol}^{-1}$ higher in energy than the local minimum. The geometry differences are small and span the whole molecule; the cation in the solid state is essentially in a low-energy molecular conformation. Molecular mechanics conformational analysis indicated that the minimum-energy conformation has an O20-C19-C12-C5 torsion angle of $-83.7^{\circ}$, compared to $109.5^{\circ}$ in the solid-state structure. The difference indicates that intermolecular interactions are significant in determining the solid-state conformation of the cation.

The analysis of the contributions to the total crystal energy using the Forcite module of Materials Studio (Dassault Systèmes, 2018) suggests that angle distortion terms are dominant in the intramolecular deformation energy and that bond

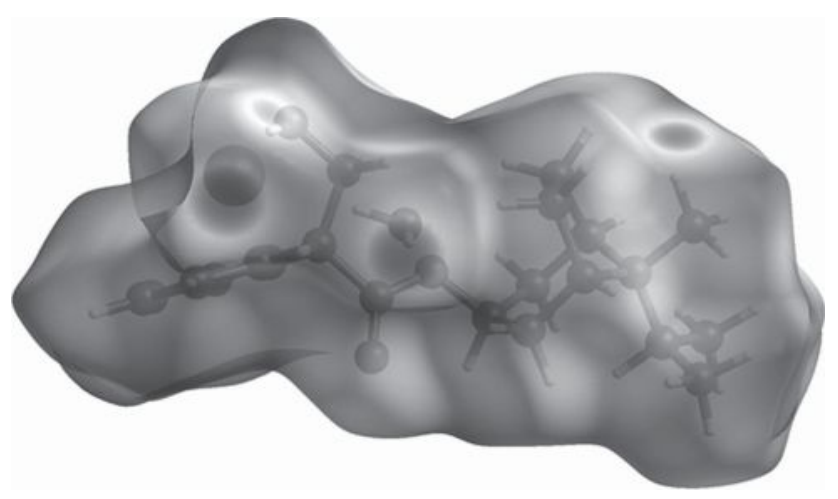

Figure 8. Hirshfeld surface of ipratropium bromide monohydrate. Intermolecular contacts longer than the sums of the van der Waals radii are colored blue, and contacts shorter than the sums of the radii are colored red. Contacts equal to the sums of radii are white. and torsion terms are also significant, as might be expected for a bicyclic ring system. The intermolecular energy is dominated by electrostatic attractions, which in this force-field-based analysis include hydrogen bonds. The hydrogen bonds are better analyzed using the results of the DFT calculation.

Hydrogen bonds are significant in the crystal structure (Table I). Both hydrogen atoms of the water molecule act as donors to the bromide cation, forming a ring with the graph set R2,4(8) (Etter, 1990; Bernstein et al., 1995; Shields et al., 2000). The hydroxyl group O17-H18 also acts as a donor to Br55. The methyl groups C39 and C43, the methylene group $\mathrm{C} 31$, and the methyne group C37 act as donors in $\mathrm{C}-\mathrm{H} \cdots \mathrm{Br}$ hydrogen bonds. Several other weaker $\mathrm{C}-$ $\mathrm{H} \cdots \mathrm{Br}$ interactions may also be present. The water molecule $\mathrm{O} 56$ acts as an acceptor in two $\mathrm{C}-\mathrm{H}$... O hydrogen bonds from the methyl groups $\mathrm{C} 43$ and $\mathrm{C} 51$. The ketone $\mathrm{O} 47$ acts as an acceptor in $\mathrm{C}-\mathrm{H}$... O hydrogen bonds from the methyl groups C33 and C39, the methylene group C31, and the methyne group $\mathrm{C} 21$ (which is intramolecular). The hydroxyl group $\mathrm{O} 17$ acts as an acceptor in a $\mathrm{C}-\mathrm{H} \cdots \mathrm{O}$ hydrogen bond from the phenyl carbon $\mathrm{C} 3$.

The volume enclosed by the Hirshfeld surface (Figure 8; Hirshfeld, 1977; Turner, et al., 2017) is $505.97 \AA^{3}, 98.51 \%$ of one-fourth the unit cell volume. The molecules are, thus, not tightly packed. All of the significant close contacts (red in Figure 8) involve the hydrogen bonds. The volume/non-H atom is relatively large, at $19.7 \AA^{3}$, reflecting the large bromide anion.

The Bravais-Friedel-Donnay-Harker (Bravais, 1866; Friedel, 1907; Donnay and Harker, 1937) morphology suggests that we might expect platy morphology for ipratropium bromide monohydrate, with $\{002\}$ as the principal faces. A texture model was not necessary, indicating that preferred orientation was not significant in this rotated capillary specimen. The powder pattern of ipratropium bromide monohydrate from this synchrotron data set is included in the Powder Diffraction File 00-066-1611.

\section{DEPOSITED DATA}

The Crystallographic Information Framework (CIF) files containing the results of the Rietveld refinement (including the raw data) and the DFT geometry optimization were 
deposited with the ICDD. The data can be requested at info@ icdd.com.

\section{ACKNOWLEDGEMENTS}

The use of the Advanced Photon Source at the Argonne National Laboratory was supported by the U.S. Department of Energy, Office of Science, Office of Basic Energy Sciences, under Contract No. DE-AC02-06CH11357. We thank Lynn Ribaud and Saul Lapidus for their assistance in the data collection, and Andrey Rogachev for the use of computing resources at IIT.

\section{FUNDING INFORMATION}

This work was partially supported by the International Centre for Diffraction Data.

\section{CONFLICTS OF INTEREST}

The authors have no conflicts of interest to declare.

Abdine, H. H., Belal, F., and Al-Badar, A. A. (2003). "Ipratropium bromide: physical properties," Profiles Drug Subst. Excip. Relat. Methodol. 30, 59-83.

Altomare, A., Cuocci, C., Giacovazzo, C., Moliterni, A., Rizzi, R., Corriero, N., and Falcicchio, A. (2013). "EXPO2013: a kit of tools for phasing crystal structures from powder data," J. Appl. Crystallogr. 46, 1231-1235.

Bernstein, J. and Zevin, L. (1992). Ipratropium Bromide Hydrate. ICDD Grant-in-Aid.

Bernstein, J., Davis, R. E., Shimoni, L., and Chang, N. L. (1995). "Patterns in hydrogen bonding: functionality and graph set analysis in crystals," Angew. Chem. Int. Ed. Engl. 34(15), 1555-1573.

Bravais, A. (1866). Etudes Cristallographiques (Gauthier Villars, Paris).

Bruno, I. J., Cole, J. C., Kessler, M., Luo, J., Motherwell, W. D. S., Purkis, L. H., Smith, B. R., Taylor, R., Cooper, R. I., Harris, S. E., and Orpen, A. G. (2004). "Retrieval of crystallographically-derived molecular geometry information," J. Chem. Inf. Sci. 44, 2133-2144.

Corrigan, D. O., Corrigan, O. I., and Healy, A. M. (2006). "Physicochemical and invitro deposition properties of salbutamol sulphate/ipratropium bromide and salbutamol sulphate/excipient spray dried mixtures for use in dry powder inhalers," Int. J. Pharm. 322, 22-30.

Dassault Systèmes (2018). Materials Studio 2019 (BIOVIA, San Diego, CA).

Donnay, J. D. H. and Harker, D. (1937). "A new law of crystal morphology extending the law of Bravais," Am. Mineral. 22, 446-447.

Dovesi, R., Orlando, R., Erba, A., Zicovich-Wilson, C. M., Civalleri, B., Casassa, S., Maschio, L., Ferrabone, M., De La Pierre, M., D-Arco, P., Noël, Y., Causà, M., and Kirtman, B. (2014). "CRYSTAL14: a program for the ab initio investigation of crystalline solids," Int. J. Quantum Chem. 114, 1287-1317.

Etter, M. C. (1990). "Encoding and decoding hydrogen-bond patterns of organic compounds," Acc. Chem. Res. 23(4), 120-126.
Favre-Nicolin, V. and Černý, R. (2002). "FOX, 'free objects for crystallography': a modular approach to ab initio structure determination from powder diffraction," J. Appl. Crystallogr. 35, 734-743.

Friedel, G. (1907). "Etudes sur la loi de Bravais," Bull. Soc. Fr. Mineral. 30 , 326-455.

Gates-Rector, S. and Blanton, T. (2019). "The powder diffraction file: a quality materials characterization database," Powder Diffr. 34(4), 352-360.

Gatti, C., Saunders, V. R., and Roetti, C. (1994). "Crystal-field effects on the topological properties of the electron-density in molecular crystals - the case of urea," J. Chem. Phys. 101, 10686-10696.

Groom, C. R., Bruno, I. J., Lightfoot, M. P., and Ward, S. C. (2016). "The Cambridge structural database," Acta Crystallogr. Sect. B: Struct. Sci., Cryst. Eng. Mater. 72, 171-179.

Hirshfeld, F. L. (1977). "Bonded-atom fragments for describing molecular charge densities," Theor. Chem. Acta. 44, 129-138.

Kaduk, J. A., Crowder, C. E., Zhong, K., Fawcett, T. G., and Suchomel, M. R. (2014). "Crystal structure of atomoxetine hydrochloride (Strattera), $\mathrm{C}_{17} \mathrm{H}_{22} \mathrm{NOCl}$," Powder Diffr. 29(3), 269-273.

Lee, P. L., Shu, D., Ramanathan, M., Preissner, C., Wang, J., Beno, M. A., Von Dreele, R. B., Ribaud, L., Kurtz, C., Antao, S. M., Jiao, X., and Toby, B. H. (2008). "A twelve-analyzer detector system for highresolution powder diffraction," J. Synch. Rad. 15(5), 427-432.

Macrae, C. F., Bruno, I. J., Chisholm, J. A., Edington, P. R., McCabe, P., Pidcock, E., Rodriguez-Monge, L., Taylor, R., van de Streek, J., and Wood, P. A. (2008). "Mercury CSD 2.0 - new features for the visualization and investigation of crystal structures," J. Appl. Crystallogr. 41, 466470.

O’Boyle, N., Banck, M., James, C. A., Morley, C., Vandermeersch, T., and Hutchison, G. R. (2011). "Open Babel: an open chemical toolbox," J. Chem. Informatics. 3, 33. doi:10.1186/1758-2946-3-33.

Peintinger, M. F., Vilela Oliveira, D., and Bredow, T. (2013). "Consistent Gaussian basis sets of triple-zeta valence with polarization quality for solid-state calculations," J. Comput. Chem. 34, 451-459.

Shields, G. P., Raithby, P. R., Allen, F. H., and Motherwell, W. S. (2000). "The assignment and validation of metal oxidation states in the Cambridge structural database," Acta Cryst. Sec. B: Struct. Sci. 56(3), 455-465.

Sykes, R. A., McCabe, P., Allen, F. H., Battle, G. M., Bruno, I. J., and Wood, P. A. (2011). "New software for statistical analysis of Cambridge structural database data,” J. Appl. Crystallogr. 44, 882-886.

Toby, B. H. and Von Dreele, R. B. (2013). "GSAS II: the genesis of a modern open source all purpose crystallography software package," J. Appl. Crystallogr. 46, 544-549.

Turner, M. J., McKinnon, J. J., Wolff, S. K., Grimwood, D. J., Spackman, P. R., Jayatilaka, D., and Spackman, M. A. (2017). CrystalExplorer17 (University of Western Australia). Available at: http://hirshfeldsurface.net.

van de Streek, J. and Neumann, M. A. (2014). "Validation of molecular crystal structures from powder diffraction data with dispersion-corrected density functional theory (DFT-D)," Acta Cryst. Sect. B: Struct. Sci., Cryst. Eng. Mater. 70(6), 1020-1032.

Wang, J., Toby, B. H., Lee, P. L., Ribaud, L., Antao, S. M., Kurtz, C., Ramanathan, M., Von Dreele, R. B., and Beno, M. A. (2008). "A dedicated powder diffraction beamline at the Advanced Photon Source: commissioning and early operational results," Rev. Sci. Instrum. 79, 085105.

Wavefunction, Inc. (2018). Spartan '18 Version 1.2.0 (Wavefunction Inc., Irvine, CA). 